\title{
Relativistic Lamé functions revisited
}

\author{
S N M Ruijsenaars \\ Centre for Mathematics and Computer Science, PO Box 94079, 1090 GB Amsterdam, \\ The Netherlands \\ Received 1 May 2001 \\ Published 23 November 2001 \\ Online at stacks.iop.org/JPhysA/34/10595
}

\begin{abstract}
A basis for the eigenfunctions of the relativistic elliptic two-particle CalogeroMoser Hamiltonian is known for a dense set in the natural parameter space. We study the question whether an interpolating basis exists, employing an asymptotic power series ansatz. For the hyperbolic specialization we determine all of the coefficients explicitly, which gives rise to formal interpolating eigenfunctions. For the elliptic case we also need a power series ansatz for the eigenvalues. We obtain the first few coefficients explicitly, thus obtaining evidence for the existence of interpolating formal eigenfunctions and eigenvalues.
\end{abstract}

PACS numbers: 02.30.Gp, 02.30.Lt

\section{Introduction}

The results presented in this paper primarily concern ordinary linear second-order analytic difference equations with special coefficient functions, and solutions with certain symmetry properties. Specifically, we deal with explicitly given elliptic coefficients, and (in greater detail) with their hyperbolic specialization, obtaining some results going beyond those in our previous papers $[1,2]$.

At present, there is a conspicuous lack of knowledge concerning the general theory for this type of equation. Explicitly worked-out special cases may yield guidance into this largely uncharted territory. In particular, they give rise to natural questions concerning more general classes of equations. On the one hand, for any question that has already been answered explicitly for the special cases, one can ask whether similar answers hold true in a more general setting. But on the other hand, an approach that does not involve detailed special function knowledge may yield existence results that would be crucial to the study of open questions in the more specialized context.

With an eye on this eventual interplay between the special and the general, we try and introduce the kind of issues we address in our special case via a stepwise narrowing of focus, imentioning pertinent questions (and a few answers) at the point where they first arise. (See also section 2 of our recent lecture notes [3] for a related account.) 
We start from an analytic difference equation (henceforth $A \Delta E$ ) of the form

$$
F(z-\mathrm{i} a)+C(z) F(z+\mathrm{i} a)=E F(z) \quad a \in(0, \infty) \quad E \in \mathbb{C} .
$$

Here, the coefficient $C(z)$ is assumed to belong to the function field

$$
\mathcal{M} \equiv\{f(z) \mid f \text { meromorphic on } \mathbb{C}\} \text {. }
$$

Moreover, we focus attention on solutions $F \in \mathcal{M}$ to (1.1). Obviously, in case $C(z)$ vanishes identically, the general solution to $(1.1)$ (with $\left.E \in \mathbb{C}^{*}\right)$ is given by $\exp (\mathrm{i} \ln (E) z / a) \mu(z)$, where $\mu(z)$ belongs to the field of $\mathrm{i} a$-periodic meromorphic functions

$$
\mathcal{P}_{\mathrm{i} a} \equiv\{\mu \in \mathcal{M} \mid \mu(z+\mathrm{i} a)=\mu(z)\} .
$$

Assuming from now on

$$
C(z) \in \mathcal{M}^{*}
$$

let $F_{1}, F_{2} \in \mathcal{M}^{*}$ be two solutions. Then their Casorati determinant

$$
\mathcal{D}\left(F_{1}, F_{2} ; z\right) \equiv F_{1}(z+\mathrm{i} a / 2) F_{2}(z-\mathrm{i} a / 2)-F_{1}(z-\mathrm{i} a / 2) F_{2}(z+\mathrm{i} a / 2)
$$

vanishes identically iff $F_{1} / F_{2} \in \mathcal{P}_{\mathrm{i} a}$. Assuming $F_{1} / F_{2} \notin \mathcal{P}_{\mathrm{i} a}$, one easily checks that (1.5) solves the first-order $\mathrm{A} \triangle \mathrm{E}$

$$
\frac{\mathcal{D}(z+\mathrm{i} a / 2)}{\mathcal{D}(z-\mathrm{i} a / 2)}=\frac{1}{C(z)} \text {. }
$$

Now suppose $F_{3}(z)$ solves (1.1), too. Then we have

$$
\mu_{j}(z) \cong \mathcal{D}\left(F_{j}, F_{3} ; z+\mathrm{i} a / 2\right) / \mathcal{D}\left(F_{1}, F_{2} ; z+\mathrm{i} a / 2\right) \in \mathcal{P}_{\mathrm{i} a} \quad j=1,2 .
$$

(By (1.6), quotients of Casorati determinants are ia-periodic.) It is straightforward to verify the identity

$$
F_{3}(z)=\mu_{1}(z) F_{2}(z)-\mu_{2}(z) F_{1}(z)
$$

Obviously, all functions of this form with $\mu_{1}, \mu_{2} \in \mathcal{P}_{\mathrm{i} a}$ solve (1.1), so the solution space may be viewed as a two-dimensional vector space over the field $\mathcal{P}_{\mathrm{i} a}$ of $\mathrm{i} a$-periodic meromorphic
functions.

Next, taking existence of a solution basis as just considered for granted, consider two A $\triangle$ Es of the above type

$\boldsymbol{F}\left(z-\mathrm{i} a_{-\delta}\right)+C_{\delta}(z) F\left(z+\mathrm{i} a_{-\delta}\right)=E_{\delta} F(z) \quad a_{\delta} \in(0, \infty) \quad E_{\delta} \in \mathbb{C} \quad \delta=+,-$.

A joint solution to these equations can be viewed as a joint eigenfunction of the two analytic difference operators $\mathcal{A}_{\delta}: \mathcal{M} \rightarrow \mathcal{M}$ given by

with

$$
\mathcal{A}_{\delta} \equiv T_{\mathrm{i} a_{-\mathrm{d}}}+C_{\delta}(z) T_{-\mathrm{i} a_{-\delta}} \quad \delta=+,-
$$

$$
\left(T_{\alpha} F\right)(z) \equiv F(z-\alpha) \quad \alpha \in \mathbb{C}^{*}
$$

It is, therefore, also a zero-eigenvalue eigenfunction of the commutator $\left[\mathcal{A}_{+}, \mathcal{A}_{-}\right]$. $a_{-}$belong to $\mathcal{M}^{*}$. For rational $a_{+} / a_{-}$solutions exist whose Casorati determinants w.r.t. $a_{+}$and since one can allow multiplie $a_{+} / a_{-}$, one still obtains an infinite-dimensional solution space, one has

$\mathcal{P}_{i} \cap \mathcal{P}$

one would expect that the joint solution space is two dimensional for irrational $a_{+} / a_{\text {. }}$. 
Of course, one should first of all prove that two solutions with the above properties existwhich is far from clear. Assuming only existence of two joint solutions $F_{+}, F_{-} \in \mathcal{M}^{*}$ for $a_{+} / a_{-}$ irrational, however, there is a simple extra condition entailing that the solution space is two dimensional with basis $\left\{F_{+}, F_{-}\right\}$: one need only assume

$$
\lim _{\lim z \rightarrow \infty} F_{+}(z) / F_{-}(z)=0
$$

for all $\operatorname{Re} z$ in some interval $I=[a, b]$.

The sufficiency of this condition can be seen as follows. (Our reasoning is adapted from the proof of theorem B.1 in [1], which deals with the special elliptic case that will be reconsidered below.) Denoting $F_{+} / F_{-}$by $Q$, it follows from (1.13) that $Q(z)$ is neither i $a_{+}$-periodic nor $\mathrm{i} a_{-}$-periodic. Thus the Casorati determinants of $F_{+}$and $F_{-}$w.r.t. $\mathrm{i} a_{+}$and $\mathrm{i} a_{-}$do not vanish identically. Now let $F$ be another solution to the joint eigenvalue equation. Then we have

$$
F(z)=\lambda_{+, \delta}(z) F_{+}(z)+\lambda_{-, \delta}(z) F_{-}(z) \quad \delta=+,-
$$

with $\lambda_{\tau \delta} \in \mathcal{P}_{\mathbf{i} a_{\delta}}, \tau, \delta=+,-$. From this we deduce

$$
\lambda_{-+}(z)-\lambda_{--}(z)=\left[\lambda_{+-}(z)-\lambda_{++}(z)\right] Q(z) .
$$

Now the functions $\lambda_{\tau \delta}(z)$ are analytic on the lines $\operatorname{Re} z=\rho \in[a, b]$, save for finitely many $\rho$. (This follows from meromorphicity and periodicity.) Fixing $\rho_{0} \in[a, b]$ such that the functions $\lambda_{\tau \delta}$ are analytic on $\operatorname{Re} z=\rho_{0}$, it follows from periodicity that they are bounded as well. From (1.15) we then get

$$
\lim _{k \rightarrow \infty} \lambda_{-+}\left(\rho_{0}+i k a_{-}\right)=\lambda_{--}\left(\rho_{0}\right) \quad k \in \mathbb{N} .
$$

Finally, we use $\lambda_{-+} \in \mathcal{P}_{\mathrm{i} a_{+}}$and our assumption $a_{+} / a_{-} \notin \mathbb{Q}$ to deduce that $\lambda_{-+}(z)$ equals $\lambda_{--}\left(\rho_{0}\right)$ for $\operatorname{Re} z=\rho_{0}$, hence for all $z$. Thus we obtain $\lambda_{\tau \delta}(z)=c_{\tau}, \tau, \delta=+,-$, so that $F$ is a linear combination of $F_{+}$and $F_{-}$, as advertised.

Let us next specialize to a situation in which $\mathcal{A}_{+}$and $\mathcal{A}_{-}$obviously commute, namely,

$$
C_{\delta}(z) \in \mathcal{P}_{\mathrm{i} a_{\delta}}^{*} \quad \delta=+,-
$$

In this setting the question concerning joint eigenfunctions is especially natural. Even so, it appears to be wide open.

Assuming existence of a joint solution $F \in \mathcal{M}^{*}$, however, one arrives at three distinct solutions $F(z), F\left(z \pm \mathrm{i} a_{+}\right)$to the $\mathrm{A} \Delta \mathrm{E} \mathcal{A}_{+} F=E_{+} F$ (since $C_{+}(z)$ is i $a_{+}$-periodic). Therefore one can write $F\left(z-\mathrm{i} a_{+}\right)$as a linear combination of $F\left(z+\mathrm{i} a_{+}\right)$and $F(z)$ with i $a_{-}$-periodic coefficients, in accordance with the second $\mathrm{A} \Delta \mathrm{E} \mathcal{A} F=E_{-} F$ obeyed by $F$. The latter $\mathrm{A} \Delta \mathrm{E}$, then, may be viewed as a constraint of monodromy type.

Specializing further, let us view $a_{+}, a_{-}$as parameters varying over $(0, \infty)$, on which $C_{+}(z)$ and $E_{+}$depend in a real-analytic way. Then we may define $C_{-}(z)$ and $E_{-}$by requiring that they be equal to $C_{+}(z)$ and $E_{+}$with $a_{+}$and $a_{-}$interchanged, respectively. In this restricted context, it is natural to search for solutions to $\mathcal{A}_{+} F=E_{+} F$ that also depend real-analytically on $a_{+}, a_{-}$, and that are invariant under the interchange of $a_{+}$and $a_{-}$. Indeed, this entails they also solve $\mathcal{A}_{-} F=E_{-} F$.

In our concrete settings there are a few more ingredients playing a role. First, we are dealing with a coefficient $C_{+}(z)$ that depends on an extra parameter $b$ in an entire fashion; since $C_{-}(z)$ is obtained by interchanging $a_{+}$and $a_{-}$, it has the same type of dependence. Second, specializing to the state of affairs in the hyperbolic case, the eigenvalue pair $\left(E_{+}, E_{-}\right)$ depends on a number in the (open) right half plane, in the sense that only for this one-parameter family and special $b$-values do we have a joint solution basis with all of the aforementioned features available. Specifically, this is the case for $b$ of the form $k a_{+}+l a_{-}, k, l \in \mathbb{Z}$. (In the elliptic case there are some further restrictions, $\mathrm{cf}[1]$.) 
Fixing $a_{+}, a_{-}$with $a_{+} / a_{-}$irrational, the numbers $b=k a_{+}+l a_{-}$are clearly dense in $\mathbb{R}$. The natural question that arises is, therefore, whether the two basis functions for the joint solution space admit an interpolation to arbitrary real $b$. Once again, this is a question that can be asked in the more general settings delineated above. However, our new results make plausible that such an interpolation may only be possible at the expense of allowing non-meromorphic solutions. Indeed, they suggest (but do not quite prove) that in our case interpolating basis functions have a natural boundary on the imaginary $z$-axis.

Let us now detail the hyperbolic coefficient functions we are concerned with in section 2 . They are given by

$$
C_{\delta}(z) \equiv \frac{\sinh \left(\pi(z+\mathrm{i} b) / a_{\delta}\right) \sinh \left(\pi\left(z-\mathrm{i} b+\mathrm{i} a_{-\delta}\right) / a_{\delta}\right)}{\sinh \left(\pi z / a_{\delta}\right) \sinh \left(\pi\left(z+\mathrm{i} a_{-\delta}\right) / a_{\delta}\right)} \quad \delta=+,-
$$

The $\mathrm{A} \Delta \mathrm{Os} \mathcal{A}_{\delta}(1.10)$ are then related to the $\mathrm{A} \Delta \mathrm{Os}$

$$
A_{\delta} \equiv \frac{\sinh \left(\pi(z-\mathrm{i} b) / a_{\delta}\right)}{\sinh \left(\pi z / a_{\delta}\right)} T_{\mathrm{i} a_{-\delta}}+(\mathrm{i} \rightarrow-\mathrm{i}) \quad \delta=+,-
$$

employed in [2] by a similarity transformation involving the 'generalized Harish-Chandra function'

$$
c\left(a_{+}, a_{-}, b ; z\right) \equiv \frac{G\left(a_{+}, a_{-} ; z-\mathrm{i} b+\mathrm{i}\left(a_{+}+a_{-}\right) / 2\right)}{G\left(a_{+}, a_{-} ; z+\mathrm{i}\left(a_{+}+a_{-}\right) / 2\right)} .
$$

(Here, $G\left(a_{+} a_{-} ; z\right)$ is the hyperbolic gamma function from [4], also known as the double sine function.) Specifically, one has

$$
\mathcal{A}_{\delta}=c(z)^{-1} A_{\delta} c(z) \quad \delta=+,-
$$

on account of the A $\triangle E$ s satisfied by the $G$-function.

Starting from $A_{\delta}$-eigenfunctions $\Psi( \pm z, y)$ with eigenvalues

and asymptotics

$$
E_{\delta}(y)=2 \cosh \left(a_{-\delta} y\right) \quad \delta=+,-
$$

$$
\Psi( \pm z, y) \sim c( \pm z) \exp ( \pm \mathrm{i} z y) \quad y \rightarrow \infty
$$

we therefore obtain $\mathcal{A}_{\delta}$-eigenfunctions

$$
F_{ \pm}(z, y) \equiv c(z)^{-1} \Psi( \pm z, y) f(y)
$$

with eigenvalues $E_{\delta}(y)$. The function $f(y)$ is at our disposal. It will be chosen such that the eigenfunctions $F_{ \pm}(z, y)$ have a certain symmetry property ('self-duality'). Moreover, it has
asymptotics

so that

$$
f(y) \sim 1 \quad y \rightarrow \infty
$$

$$
F_{+}(z, y) \sim \exp (i z y) \quad y \rightarrow \infty .
$$

The elliptic coefficient functions at issue in section 3 are given by

Here we have

$$
C_{\delta}(z) \equiv \exp (-2 b r) \frac{s_{\delta}(z+\mathrm{i} b) s_{\delta}\left(z-\mathrm{i} b+\mathrm{i} a_{-\delta}\right)}{s_{\delta}(z) s_{\delta}\left(z+\mathrm{i} a_{-\delta}\right)} \quad \delta=+,-
$$

$$
s_{\delta}(z) \equiv s\left(r, a_{8} ; z\right) \quad \delta=+,-
$$

and $s(r, a ; z)$ is defined in terms of the Weierstrass $\sigma$-function $\sigma\left(z ; \omega, \omega^{\prime}\right)$ by

$$
s(r, a ; z) \equiv \sigma\left(z ; \frac{\pi}{2 r}, \frac{i a}{2}\right) \exp \left(-\eta z^{2} r / \pi\right)
$$


Thus $C_{\delta}(z)$ is an elliptic function with periods $\pi / r, \mathrm{i} a_{\delta}$.

The elliptic $\mathrm{A} \Delta \mathrm{Os} \mathcal{A}_{\delta}$ are related to the $\mathrm{A} \Delta \mathrm{O}$ s

$$
A_{\delta} \equiv \exp (-b r) \frac{s_{\delta}(z-\mathrm{i} b)}{s_{\delta}(z)} T_{\mathrm{i} a_{-\delta}}+(\mathrm{i} \rightarrow-\mathrm{i}) \quad \delta=+,-
$$

employed in [1] by the similarity transformation (1.21), with $c(z)$ now given by

$$
c\left(r, a_{+}, a_{-}, b ; z\right) \equiv \frac{G\left(r, a_{+}, a_{-} ; z-\mathrm{i} b+\mathrm{i}\left(a_{+}+a_{-}\right) / 2\right)}{G\left(r, a_{+}, a_{-} ; z+\mathrm{i}\left(a_{+}+a_{-}\right) / 2\right)} .
$$

Here, $G\left(r, a_{+} a_{-} ; z\right)$ is the elliptic gamma function introduced in [4].

At the elliptic level, too, there exist, for suitable parameters $a_{+}, a_{-}, b$, joint eigenfunctions $\Psi( \pm z, y)$ of the A $\Delta \mathrm{Os} A_{\delta}$ with eigenvalues $E_{\delta}(y)$ satisfying

$$
E_{\delta}(y) \sim \exp \left(a_{-\delta} y\right) \quad y \rightarrow \infty \quad \delta=+,-
$$

and asymptotics (1.23) (cf equations $(1.22)-(1.26)$ in [1]). Thus they give rise to $\mathcal{A}_{\delta}$ eigenfunctions $F_{ \pm}(z, y)$ (1.24) with asymptotics (1.26) whenever $f(y)$ satisfies (1.25).

Let us now sketch the contents of section 2 , which deals with the hyperbolic case. In section 2.1 we introduce and explore a power series ansatz for a solution to the $A \Delta E s$ $\mathcal{A}_{\delta} F=2 \cosh \left(a_{-\delta} y\right) F$ with asymptotics $\exp (\mathrm{i} z y)$ for $y \rightarrow \infty$. This yields a system of A $\triangle$ Es coupling the coefficients. The requirement that the coefficients vanish for $z \rightarrow \infty$ gives rise to a special type of solution that is unique when it exists.

In section 2.2 we exploit results from our paper [2] to prove that this special type of solution does exist for $a_{+} / a_{-}$irrational and $b$ of the form $\left(N_{+}+1\right) a_{+}-N_{-} a_{-}, N_{+}, N_{-} \in \mathbb{N}$. In section 2.3 we obtain the coefficients explicitly. We show they admit a real-analytic extension to real $b$ by using the $q$-binomial theorem. In this way we obtain formal interpolating $\mathcal{A}_{8}$-eigenfunctions. Though we discuss them in some detail, we leave various questions open.

The elliptic case is studied in section 3, following the pattern laid out for the hyperbolic case. Here, however, we cannot go very far. Indeed, our results are only some small steps towards a complete resolution of the interpolation issue. But just as in the hyperbolic case, they do supply some new ideas for further studies.

To conclude this introduction, let us add that our hyperbolic results can be exploited to obtain arbitrary- $b$ (non-formal) trigonometric eigenfunctions. (In section 4 of [2] we only handled a discrete set of $b$-values.) To keep this paper within bounds, we will return to the trigonometric case elsewhere.

\section{The hyperbolic regime}

\subsection{A power series ansatz}

Consider the eigenvalue equations

$$
\left(\mathcal{A}_{-\delta} F\right)(z, y)=2 \cosh \left(a_{\delta} y\right) F(z, y) \quad a_{\delta} \in(0, \infty) \quad \delta=+,-
$$

with $\mathcal{A}_{-\delta}$ given by (1.10) and (1.18). Since the coefficients $C_{\delta}(z)(1.18)$ tend to 1 exponentially as $\operatorname{Re} z \rightarrow \infty$, one may expect that solutions $F_{+}(z, y)$ exist with plane wave asymptotics $\exp (\mathrm{izy})$ as $\operatorname{Re} z \rightarrow \infty$. But in fact we are going to impose the 'dual' requirement

$$
F_{+}(z, y) \sim \exp (\mathrm{i} z y) \quad \operatorname{Re} y \rightarrow \infty .
$$

Mọre generally, requiring

$$
a_{+} / a_{-} \notin \mathbb{Q}
$$


until further notice, we are going to study the power series ansatz

$$
F_{+}(z, y)=\mathrm{e}^{\mathrm{i} z y} \sum_{k, l=0}^{\infty} c_{k l}(z) \exp \left(-2 k a_{+} y\right) \exp \left(-2 l a_{-} y\right) \quad c_{00}(z) \equiv 1 .
$$

(Note that (2.3) is necessary for all monomials to be distinct.)

To be sure, this ansatz may appear unmotivated at this point. Its relevance is, however, suggested by our previous results in [2]. We begin by studying it so as to make clear how the eigenfunctions from [2] fit in this general framework, unencumbered by the detailed formulae presented later on. Furthermore, proceeding in this reverse order will be advantageous in our study of the elliptic case in section 3.

Plugging (2.4) into (2.1), we obtain

$$
\begin{aligned}
\sum_{k, l=0}^{\infty} \mathrm{e}^{-2\left(k a_{+}+l a_{.}\right) y}\left[c_{k l}\left(z-\mathrm{i} a_{\delta}\right)+C_{-\delta}(z) \mathrm{e}^{-2 a_{\delta} y} c_{k l}\left(z+\mathrm{i} a_{\delta}\right)\right] & \\
= & \left(1+\mathrm{e}^{-2 a_{\delta} y}\right) \sum_{k, l=0}^{\infty} c_{k l}(z) \mathrm{e}^{-2\left(k a_{+}+l a_{-}\right) y} \quad \delta=+,-.
\end{aligned}
$$

Comparing coefficients, we get for $\delta=+$

$c_{m n}\left(z-\mathrm{i} a_{+}\right)-c_{m n}(z)=-C_{-}(z) c_{m-1, n}\left(z+\mathrm{i} a_{+}\right)+c_{m-1, n}(z) \quad m, n \in \mathbb{N}$

and for $\delta=-$

$c_{m n}\left(z-\mathrm{i} a_{-}\right)-c_{m n}(z)=-c_{+}(z) c_{m, n-1}\left(z+\mathrm{i} a_{-}\right)+c_{m, n-1}(z) \quad m, n \in \mathbb{N}$.

Here, we have boundary conditions

$$
c_{00} \equiv 1 \quad c_{-1, l} \equiv 0 \quad l \in \mathbb{N} \quad c_{k,-1} \equiv 0 \quad k \in \mathbb{N} .
$$

We proceed by analysing the system $(2.6)-(2.8)$ of coupled linear $A \Delta E s$. First, let us note that the eigenvalue equations $(2.1)$ do not fix the dependence on the spectral variable multiply (2.4) by an arbitrary form exists, it is highly non-unique. In particular, we can

$$
p(y) \equiv \sum_{m, n=0}^{\infty} \alpha_{m n} \exp \left(-2\left(m a_{+}+n a_{-}\right) y\right) \quad \alpha_{00}=1
$$

to obtain another formal solution with coefficients

$$
c_{k l}^{(p)}(z)=\sum_{i=0}^{k} \sum_{j=0}^{l} c_{i j}(z) \alpha_{k-i, l-j}
$$

Then the coefficients $c_{m n}^{(p)}$

To discard this ambiguity,

$$
c_{k !}(z) \rightarrow 0 \quad \operatorname{Re} z \rightarrow \infty \text { sufficient to require }
$$

Of course, at this stage it is far from $\quad \operatorname{Re} z \rightarrow \infty \quad \forall(k, l) \in \mathbb{N}^{2} \backslash\{(0,0)\}$.

so, let us assume that a solution tom clear that a solution with this asymptotics exists. Even precise, we assume that for arbitrary $m=0$ subsystem satisfying (2.11) exists. To be quite functions $\alpha_{n}\left(a_{+}, a_{-}, b ; z\right), n \in \mathbb{N}^{*}$, such that $a_{+}, a_{-}$satisfying (2.3) and real $b$ there exist $\alpha_{n}(z) \in \mathcal{P}_{\text {ia. }} . \quad \forall n \in \mathbb{N}^{*}$

$$
\begin{array}{ll}
\alpha_{n}\left(z-\mathrm{i} a_{-}\right)-\alpha_{n}(z)=-C_{+}(z) \alpha_{n-1}\left(z+\mathrm{i} a_{-}\right)+\alpha_{n-1}(z) & \forall n \in \mathbb{N}^{*} \quad \alpha_{0}(z) \equiv 1 \\
\alpha_{n}(z) \rightarrow 0 \quad \operatorname{Re} z \rightarrow \infty \quad \forall n \in \mathbb{N}^{*}
\end{array}
$$


This existence assumption will be shown to be non-vacuous later on. (Indeed, we will exhibit the $\alpha_{n}$ explicitly.) But for now we only derive some illuminating consequences. First, we claim our assumption entails that the system (2.6)-(2.8) admits a unique solution with asymptotics (2.11).

We prove this claim in several steps. To begin with, we show that the above functions $\alpha_{n}$ are uniquely determined by (2.12)-(2.14). Indeed, taking first $n=1$, the difference of two such functions is $\mathrm{i} a_{+}$-periodic and $\mathrm{i} a_{-}$-periodic, and it vanishes for $\operatorname{Re} z \rightarrow \infty$. But then it vanishes identically, $\mathrm{cf}(1.12)$. Proceeding inductively, this yields the desired uniqueness.

Next, we assert that the coefficients

$$
c_{m n}\left(a_{+}, a_{-}, b ; z\right) \equiv \alpha_{m}\left(a_{-}, a_{+}, b ; z\right) \alpha_{n}\left(a_{+}, a_{-}, b ; z\right) \quad m, n \in \mathbb{N}
$$

solve the system (2.6)-(2.8) and have asymptotics (2.11). The last assertion is immediate from (2.14). To prove the solution property, we recall that (2.12), (2.13) amount to $c_{0 n}$ solving the $m=0$ subsystem. Since $C_{-}(z)$ is obtained from $C_{+}(z)$ by interchanging $a_{+}$and $a_{-}$, the coefficients $c_{m 0}$ satisfy the $n=0$ subsystem. Using now i $a_{\delta}$-periodicity, the solution property for the general system easily follows.

Finally, to prove the uniqueness of this solution, we need only invoke the argument showing uniqueness of the functions $\alpha_{n}$. Indeed, the same reasoning shows that the solution $c_{m 0}$ to the $n=0$ subsystem with the prescribed asymptotics is unique. Then we successively get uniqueness of $c_{11}, c_{12}, c_{13}, \ldots, c_{21}, c_{22}, \ldots$ etc. Hence our claim is proved.

We continue with a few more consequences of our existence assumption. First, it entails the symmetry property

$$
\alpha_{n}\left(a_{+}, a_{-}, b ; z\right)=\alpha_{n}\left(a_{+}, a_{-}, a_{+}+a_{-}-b ; z\right) \quad n \in \mathbb{N} .
$$

To see this, note that the function $C_{+}(z)(1.18)$ is invariant under $b \rightarrow a_{+}+a_{-}-b$. Therefore, (2.16) follows from uniqueness of the functions $\alpha_{n}$ with the properties (2.12)(2.14).

Combining (2.16) and (2.15), we now obtain

$$
\begin{aligned}
& c_{k l}\left(a_{+}, a_{-}, b ; z\right)=c_{l k}\left(a_{-}, a_{+}, b ; z\right) \\
& c_{k l}\left(a_{+}, a_{-}, b ; z\right)=c_{k l}\left(a_{+}, a_{-}, a_{+}+a_{-}-b ; z\right) .
\end{aligned}
$$

Thus the corresponding formal solution

$$
F_{+}\left(a_{+}, a_{-}, b ; z, y\right) \equiv \exp (\mathrm{i} z y) \sum_{k, l=0}^{\infty} c_{k l}\left(a_{+}, a_{-}, b ; z\right) \exp \left(-2\left(k a_{+}+l a_{-}\right) y\right)
$$

to the eigenvalue equations (2.1) satisfies

$$
\begin{aligned}
& F_{+}\left(a_{+}, a_{-}, b ; z, y\right)=F_{+}\left(a_{-}, a_{+}, b ; z, y\right) \\
& F_{+}\left(a_{+}, a_{-}, b ; z, y\right)=F_{+}\left(a_{+}, a_{-}, a_{+}+a_{-} b ; z, y\right) .
\end{aligned}
$$

It is not a simple matter to find the unique functions $\alpha_{n}$ obeying (2.12)-(2.14) directly. Here we only determine $\alpha_{1}$ (which is easy), and then exploit results from [2] to obtain all of the $\alpha_{n}$.

Thus, consider the $n=1 \mathrm{~A} \Delta \mathrm{E}(2.13)$,

$$
\alpha_{1}\left(z-i a_{-}\right)-\alpha_{1}(z)=-C_{+}(z)+1 \text {. }
$$

Using (1.18), this can be rewritten as

$$
\begin{aligned}
& \alpha_{1}\left(z-\mathrm{i} a_{-}\right)-\alpha_{1}(z)=\gamma\left(a_{+}, a_{-}, b\right)\left[\operatorname{coth}\left(\pi z / a_{+}\right)-\operatorname{coth}\left(\pi\left(z+\mathrm{i} a_{-}\right) / a_{+}\right)\right] \\
& \gamma\left(a_{+}, a_{-}, b\right) \equiv \sinh \left(\mathrm{i} \pi\left(b-a_{-}\right) / a_{+}\right) \sinh \left(\mathrm{i} \pi b / a_{+}\right) / \sinh \left(\mathrm{i} \pi a_{-} / a_{+}\right) .
\end{aligned}
$$


Hence we immediately obtain the solution

$$
\alpha_{1}\left(a_{+}, a, b ; z\right)=\gamma\left(a_{+}, a_{-}, b\right)\left[\operatorname{coth}\left(\pi\left(z+\mathrm{i} a_{-}\right) / a_{+}\right)-1\right] .
$$

Note that it is indeed invariant under $b \rightarrow a_{+}+a_{-}-b$, and that it is ill defined for $a_{+}=a_{-}$.

To solve the $n=2$ equations directly is not a routine matter. (Moreover, we should repeat at this point that existence of a solution is not clear, a priori.) Thus we now turn to the pertinent results from our paper [2], which will lead to the desired solutions $\alpha_{n}\left(a_{+}, a_{-}, b ; z\right), n \in \mathbb{N}$. (They are given by (2.59) below.)

\subsection{Eigenfunctions for a dense set of $b$-values}

In section 3 of [2] we obtained joint $A_{\delta}$-eigenfunctions $M\left(a_{+}, a_{-}, b ; \pm z, p\right)$ with eigenvalues $2 \cosh \left(\pi p / a_{\delta}\right), \delta=+,-$, for all $a_{+}, a_{-} \in(0, \infty)$ and $b$ of the form $k_{+} a_{+}+k_{-} a_{-}, k_{+}, k_{-} \in \mathbb{Z}$. (For other eigenvalue pairs we have no information concerning joint $A_{\delta}$-eigenfunctions.) More specifically, the hyperbolic specialization of the elliptic $A_{\delta}$-eigenfunction $\Psi(z, y)$ from [1] (already mentioned in the introduction) is related to $M(z, p)$ via

$$
\Psi\left(z, \pi p / a_{+} a_{-}\right)=M(z, p) w(p)^{1 / 2}
$$

where $w(p)$ is the hyperbolic weight function from [4].

In this connection we recall that the dependence of the joint eigenfunctions on the spectral variable is a matter of convention. As it turns out, in the hyperbolic case this dependence can be chosen in such a way that one obtains joint eigenfunctions that are invariant under interchanging $z$ and $p$. Indeed, the functions $M( \pm z, p)$ have this striking 'self-duality' property, cf equations (3.71), (3.72) in [2].

We proceed by defining $\mathcal{A}_{\delta}$-eigenfunctions

where

$$
F_{ \pm}(z, y) \equiv \mathrm{e}^{\mathrm{i} \phi} M( \pm z, p) / c(z) c(p) \quad p=a_{+} a_{-} y / \pi
$$

$$
\phi \equiv \frac{\pi}{2}\left[\left(1-b / a_{+}\right)\left(1-b / a_{-}\right)-1\right] .
$$

When one views these functions as functions of $z$ and $p$, they are by construction self-dual as the two regimes at issue. Clearly, one has in the context of this paper it is expedient to employ the same spectral variable $y$ for

$$
F_{-}(z, y)=-u(-z) F_{+}(-z, y)
$$

$$
u(z) \equiv-c(z) / c(-z)
$$

is the $u$-function (' $S$-matrix') from [4].

The phase (2.28) is chosen such that one gets asymptotics

$$
\begin{aligned}
& \begin{array}{ll}
F_{+}(z, y) \sim \exp (\mathrm{i} z y) & \operatorname{Re} y \rightarrow \infty \\
F_{-}(z, y) \sim-u(-z) \exp (-\mathrm{i} z y) & \operatorname{Re} y \rightarrow \infty
\end{array} \\
& \text { The two functions } F_{ \pm}(z, y) \text { are related } \quad \operatorname{Re} y \rightarrow \infty \text {. }
\end{aligned}
$$

on the imaginary $y$-axis. Restricting by $\mathrm{i} a_{+}$- or i $a_{-}$-periodic multipliers for a discrete set holds true for $\operatorname{Re} z>0$, however. For ittion to $\operatorname{Re} y>0$ from now on, the condition (1.13) the two-dimensional space of (meromorphic) function $a_{+} / a_{-}$, the functions $F_{ \pm}(z, y)$ therefore span

$$
\begin{aligned}
F\left(z-\mathrm{i} a_{\delta}\right)+ & \frac{\sinh \left(\pi\left(z+\mathrm{i} k_{\delta} a_{\delta}\right) / a_{-\delta}\right) \sinh \left(\pi\left(z-\mathrm{i}\left(k_{\delta}-1\right) a_{\delta}\right) / a_{-\delta}\right)}{\sinh \left(\pi z / a_{-\delta}\right) \sinh \left(\pi\left(z+\mathrm{i} a_{\delta}\right) / a_{-\delta}\right)} F\left(z+\mathrm{i} a_{\delta}\right) \\
& =2 \cosh \left(a_{\delta} y\right) F(z) \quad k_{\delta} \in \mathbb{Z} \quad \delta=+,-.
\end{aligned}
$$


Since our main interest in this paper concerns interpolation with (2.3) in effect, we may as well restrict attention to $b$ of the form

$$
b=\left(N_{+}+1\right) a_{+}-N_{-} a_{-} \quad N_{+}, N_{-} \in \mathbb{N} .
$$

(Indeed, these $b$-values are already dense in $\mathbb{R}$.) The pertinent $c$-function can be calculated from the $A \triangle E$ s satisfied by the hyperbolic gamma function, yielding

$c(z)=(-\mathrm{i})^{2 N_{+} N_{-}+N_{+}+N_{-}+1} \prod_{k=1}^{N_{-}} 2 \sinh \left(\pi\left(z+\mathrm{i} k a_{-}\right) / a_{+}\right) / \prod_{j=0}^{N_{+}} 2 \sinh \left(\pi\left(z-\mathrm{i} j a_{+}\right) / a_{-}\right)$.

From equation (3.65) in [2] we then obtain

$$
\begin{aligned}
F_{+}(z, y)=\prod_{\delta=+,-} q_{\delta}^{N_{\delta}\left(N_{\delta}+1\right) / 2} \prod_{j=1}^{N_{\delta}}\left[4 \sinh \left(\pi\left(z+\mathrm{i} j a_{\delta}\right) / a_{-\delta}\right) \sinh \left(\pi\left(p+\mathrm{i} j a_{\delta}\right) / a_{-\delta}\right)\right]^{-1} \\
\quad \times \prod_{\delta=++_{+}-} \exp \left(\pi N_{\delta}(z+p) / a_{-\delta}\right) S_{N_{\delta}}\left(q_{\delta} ; \exp \left(-2 \pi z / a_{-\delta}\right), \exp \left(-2 \pi p / a_{-\delta}\right)\right) \\
\quad \times \exp (\mathrm{i} z y) \quad p=a_{+} a_{-} y / \pi .
\end{aligned}
$$

Here, $q_{+}$and $q_{-}$are the phases

$$
q_{+} \equiv \exp \left(\mathbf{i} \pi a_{+} / a_{-}\right) \quad q_{-} \equiv \exp \left(\mathrm{i} \pi a_{-} / a_{+}\right)
$$

and $S_{N}(q ; r, s)$ is a polynomial in $r$ and $s$ with coefficients that are Laurent polynomials in $q$ :

$$
S_{N}(q ; r, s) \equiv \sum_{k, l=0}^{N} c_{k l}^{(N)}(q) r^{k} s^{l}
$$

The coefficients are defined by equations (2.2)-(2.5) in [2]. (Note the typo in (2.2): the second dot sequence should be omitted.) For our purposes, it is enough to use two formulae that easily follow from their definition in [2], namely,

$$
S_{N}(q ; r, 0)=q^{N(N+1) / 2} \prod_{j=1}^{N}\left(1-q^{-2 j} r\right)
$$

and

$$
\begin{aligned}
& \frac{S_{N}(q ; r, s)}{S_{N}(q ; r, 0)}=\sum_{l=0}^{N} d_{l}^{(N)}(q ; r) s^{l} \\
& d_{l}^{(N)}(q ; r)=(-)^{l} \sum_{1 \leqslant i_{1}<\cdots<i_{l} \leqslant N} q^{-2\left(i_{1}+\cdots+i_{l}\right)} \prod_{j=1}^{l} \frac{1-q^{-2 j+2(N+1)} r}{1-q^{-2 j} r}
\end{aligned}
$$

Using (2.39), (2.40) and the notation

$$
r_{\delta} \equiv \exp \left(-2 \pi z / a_{-\delta}\right) \quad s_{\delta} \equiv \exp \left(-2 a_{\delta} y\right) \quad \delta=+,-
$$

we can rewrite $(2.36)$ as

$$
\begin{aligned}
\frac{F_{+}(z, y)}{\exp (\mathrm{i} z y)} & =\prod_{\delta=+,-} q_{\delta}^{-N_{\delta}\left(N_{\delta}+1\right) / 2} S_{N_{\delta}}\left(q_{\delta} ; r_{\delta}, s_{\delta}\right) \prod_{j=1}^{N_{\delta}} \frac{1}{\left(1-q_{\delta}^{-2 j} r_{\delta}\right)\left(1-q_{\delta}^{-2 j} s_{\delta}\right)} \\
& =\prod_{\delta=+,-}\left(\sum_{l=0}^{N_{\delta}} d_{l}^{\left(N_{\delta}\right)}\left(q_{\delta} ; r_{\delta}\right) s_{\delta}^{l}\right) \prod_{j=1}^{N_{\delta}}\left(1-q_{\delta}^{-2 j} s_{\delta}\right)^{-1}
\end{aligned}
$$

From this one easily sees that the rhs can be written as a product of two power series in $s_{+}$and $s_{-}$, which converge for $\left|s_{\delta}\right|<1, \delta=+,-$. Moreover, the asymptotics $(2.31)$ is clear from $d_{0}^{(N)}=1$. 


\subsection{Formal interpolating eigenfunctions}

We are now prepared to return to our ansatz (2.4) for solving the eigenvalue equations (2.1). As we have just seen, a (non-formal) solution of this form exists for arbitrary positive $a_{+}, a_{-}$and $b$ of the form (2.34). Requiring once more (2.3) so as to ensure independence of the monomials $s_{+}^{m} s_{-}^{n}$, we may now deduce that their coefficients $c_{m n}$ satisfy the system (2.6)-(2.8).

We continue to obtain the coefficients associated with (2.43) in explicit form. First, let us note (2.41) entails

$$
\prod_{j=1}^{N_{\delta}}\left(1-q_{\delta}^{-2 j} s_{\delta}\right)=\sum_{l=0}^{N_{\delta}} d_{l}^{\left(N_{\delta}\right)}\left(q_{\delta} ; 0\right) s_{\delta}^{l} .
$$

Also, (2.37) and (2.3) yield $q_{\delta}^{l} \neq 1$ for $\delta=+,-$ and $l \in \mathbb{Z}$. Now we are going to exploit the identity

$$
d_{l}^{\left(N_{\delta}\right)}\left(q_{\delta} ; 0\right)=\prod_{j=1}^{l} \frac{1-q_{\delta}^{2 j-2\left(N_{\delta}+1\right)}}{1-q_{\delta}^{2 j}} .
$$

Its validity can be deduced directly from the recurrence relation obeyed by the sum coefficient in (2.41), cf equations (2.26) and (2.31) in [2]. But one can also view it as a special case of the $q$-binomial theorem. (This was pointed out before in [5].)

Before recalling and using the general version of this theorem, we rewrite $(2.45)$ using standard $q$-notation [6] as

$$
d_{l}^{\left(N_{\delta}\right)}\left(q_{\delta} ; 0\right)=\left(q_{\delta}^{-2 N_{\delta}} ; q_{\delta}^{2}\right)_{l} /\left(q_{\delta}^{2} ; q_{\delta}^{2}\right)_{l} .
$$

More generally, we have from (2.41)

$$
d_{l}^{\left(N_{\delta}\right)}\left(q_{\delta} ; r_{\delta}\right)=\frac{\left(q_{\delta}^{-2 N_{\delta}} ; q_{\delta}^{2}\right)_{l}}{\left(q_{\delta}^{2} ; q_{\delta}^{2}\right)_{l}} \frac{\left(q_{\delta}^{2 N_{\delta}} r_{\delta} ; q_{\delta}^{-2}\right)_{l}}{\left(q_{\delta}^{-2} r_{\delta} ; q_{\delta}^{-2}\right)_{l}}
$$

We now make the key observation that we can write

$$
q_{+}^{2 N_{+}}=\exp \left(2 \mathrm{i} \pi\left(b-a_{+}\right) / a_{-}\right) \quad q_{-}^{2 N_{-}}=\exp \left(-2 \mathrm{i} \pi b / a_{+}\right) .
$$

(Recall our standing assumption (2.34).) Thus, setting

with

$$
d_{l}^{\delta}(b, r) \equiv \frac{\left(\mathrm{e}^{\mathrm{i} \phi_{\delta}(b)} ; q_{\delta}^{2}\right)_{l}}{\left(q_{\delta}^{2} ; q_{\delta}^{2}\right)_{l}} \frac{\left(\mathrm{e}^{-\mathrm{i} \phi_{\delta}(b)} r ; q_{\delta}^{-2}\right)_{l}}{\left(q_{\delta}^{-2} r ; q_{\delta}^{-2}\right)_{l}} \quad l \in \mathbb{N} \quad \delta=+,-
$$

$$
\phi_{+}(b) \equiv 2 \pi\left(a_{+}+a_{-}-b\right) / a_{-} \quad \phi_{-}(b) \equiv 2 \pi b / a_{+}
$$

we obtain well-defined coefficients that depend on $b \in \mathbb{R}$ in a real-analytic fashion. Furthermore, they are clearly the unique coefficients with this property that satisfy

$d_{l}^{\delta}\left(\left(N_{+}+1\right) a_{+}-N_{-} a_{-}, r\right)=d_{l}^{\left(N_{s}\right)}\left(q_{\delta} ; r\right) \quad l=0, \ldots, N_{\delta} \quad \delta=+,-$.
Consider now the power series

$$
P_{\delta}\left(b, r_{\delta} ; s_{\delta}\right) \equiv \sum_{l=0}^{\infty} d_{l}^{\delta}\left(b, r_{\delta}\right) s_{\delta}^{l} .
$$

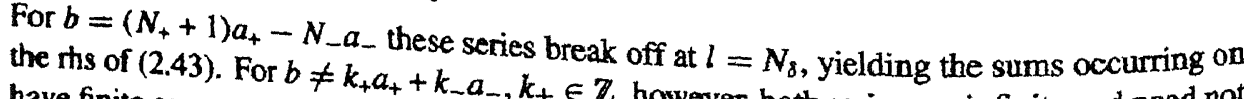
have finite convergence radius (due to $k_{ \pm} \in \mathbb{Z}$, however, both series are infinite and need not 
We do not address this convergence problem here. Instead, we continue to study the interpolation in the sense of formal power series. Using once more standard $q$-notation [6], we have

$$
P_{\delta}(b, 0 ; s)={ }_{1} \phi_{0}\left(\mathrm{e}^{\mathrm{i} \phi_{\delta}(b)} ;-; q_{\delta}^{2}, s\right) .
$$

Now we recall the $q$-binomial theorem [6]:

$$
{ }_{1} \phi_{0}(\alpha ;-; q, s) \equiv \sum_{l=0}^{\infty} \frac{(\alpha ; q)_{l}}{(q ; q)_{l}} s^{l}=\frac{(\alpha s ; q)_{\infty}}{(s ; q)_{\infty}} \quad|s|,|q|<1 .
$$

(For $\alpha=q^{-N}, N \in \mathbb{N}$, the series breaks off. Thus one may take $q$ on the unit circle, entailing the identity (2.45), as announced.) It entails

$$
\begin{aligned}
{ }_{1} \phi_{0}(\alpha ;-; q, s)^{-1} & ={ }_{1} \phi_{0}\left(\alpha^{-1} ;-; q, \alpha s\right) \\
& =\sum_{l=0}^{\infty} \frac{\left(\alpha^{-1} ; q\right)_{l}}{(q ; q)_{l}} \alpha^{l} s^{l} \quad|s|<1 \quad|q|<1 .
\end{aligned}
$$

Therefore, we can explicitly determine the reciprocals of the formal power series $P_{\delta}(b, 0 ; s)$. They read

$$
1 / P_{\delta}(b, 0 ; s)=\sum_{k=0}^{\infty} \frac{\left(\exp \left(-\mathrm{i} \phi_{\delta}(b)\right) ; q_{\delta}^{2}\right)_{k}}{\left(q_{\delta}^{2} ; q_{\delta}^{2}\right)_{k}} \exp \left(\mathrm{i} k \phi_{\delta}(b)\right) s^{k} .
$$

Putting the pieces together, we finally get the interpolating formal power series

$$
\frac{F_{+}(z, y)}{\exp (\mathrm{i} z y)}=\prod_{\delta=+,-} \sum_{m_{\delta}=0}^{\infty} c_{m_{\delta}}^{\delta}(b ; z) \exp \left(-2 m_{\delta} a_{\delta} y\right)
$$

where the coefficients are given by

$$
\begin{aligned}
c_{n}^{\delta}(b ; z)=\sum_{l=0}^{n} & \frac{\left(\exp \left(\mathrm{i} \phi_{\delta}(b)\right) ; q_{\delta}^{2}\right)_{l}}{\left(q_{\delta}^{2} ; q_{\delta}^{2}\right)_{l}} \frac{\left(\exp \left(-\mathrm{i} \phi_{\delta}(b)\right) \exp \left(-2 \pi z / a_{-\delta}\right) ; q_{\delta}^{-2}\right)_{l}}{\left(q_{\delta}^{-2} \exp \left(-2 \pi z / a_{-\delta}\right) ; q_{\delta}^{-2}\right)_{l}} \\
& \times \frac{\left(\exp \left(-\mathrm{i} \phi_{\delta}(b)\right) ; q_{\delta}^{2}\right)_{n-l}}{\left(q_{\delta}^{2} ; q_{\delta}^{2}\right)_{n-l}} \exp \left(\mathrm{i}(n-l) \phi_{\delta}(b)\right) \quad \delta=+,-.
\end{aligned}
$$

Now we recall our previous analysis of the power series ansatz (2.4). The uniqueness results we proved entail in particular that the functions $\alpha_{n}$ are given by

$$
\begin{aligned}
\alpha_{n}\left(a_{+}, a_{-} b ; z\right) & =\sum_{l=0}^{n} \frac{\left(\exp \left(2 \pi \mathrm{i} b / a_{+}\right) ; q_{-}^{2}\right)}{\left(q_{-}^{2} ; q_{-}^{2}\right)_{l}} \frac{\left(\exp \left(-2 \pi(z+\mathrm{i} b) / a_{+}\right) ; q_{-}^{-2}\right)_{l}}{\left(\exp \left(-2 \pi\left(z+\mathrm{i} a_{-}\right) / a_{+}\right) ; q_{-}^{-2}\right)_{l}} \\
& \times \frac{\left(\exp \left(-2 \pi \mathrm{i} b / a_{+}\right) ; q_{-}^{2}\right)_{n-l}}{\left(q_{-}^{2} ; q_{-}^{2}\right)_{n-l}} \exp \left(2 \pi \mathrm{i}(n-l) b / a_{+}\right)
\end{aligned}
$$

with $q_{-}=\exp \left(\mathbf{i} \pi a_{-} / a_{+}\right)$. This is the explicit formula announced above.

To conclude this section, we add a number of observations related to (2.59). We begin by noting that the only one of the three properties (2.12)-(2.14) that is obvious from (2.59) is (2.12) (i $a_{+}$-periodicity). It would be a quite unpleasant task to verify the two remaining properties directly. Note that the asymptotics (2.14) does follow from the $q$-binomial theorem. Indeed, (2.55) entails the identities

$$
\sum_{l=0}^{n} \frac{(\alpha ; q)_{l}}{(q ; q)_{l}} \frac{\left(\alpha^{-1} ; q\right)_{n-l}}{(q ; q)_{n-l}} \alpha^{n-l}=0 \quad n>0 \quad|q|<1 .
$$

Clearly, these are still valid for all $q$ on the unit circle that satisfy $q^{k} \neq 1, k \in \mathbb{N}^{*}$, which yields (2.14). 
Next, we note that the parameter symmetry (2.16) is not at all clear from (2.59). Even for

$$
\alpha_{1}\left(a_{+}, a_{-}, b ; z\right)=\frac{1-\mathrm{e}^{2 \pi \mathrm{i} b / a_{+}}}{1-\mathrm{e}^{2 \pi \mathrm{i} a_{-} / a_{+}}}\left(\frac{1-\mathrm{e}^{-2 \pi(z+\mathrm{i} b) / a_{+}}}{1-\mathrm{e}^{-2 \pi\left(z+\mathrm{i} a_{-}\right) / a_{+}}}-1\right)
$$

some effort is needed to rewrite it as (2.25), which is manifestly invariant. Note that this symmetry entails that for $b=-N_{+} a_{+}+\left(N_{-}+1\right) a_{-}$the formal power series $\mathcal{A}_{-}$-eigenfunction $F_{+}\left(a_{+}, a_{-}, b ; z, y\right)$ is equal to the (non-formal) $\mathcal{A}_{\delta}$-eigenfunction (2.36). In fact, since for $b$ of the form $\left(N_{+}+1\right) a_{+}-N_{-} a_{-}$the map $b \mapsto a_{+}+a_{-}-b$ on the functions $\alpha_{m}\left(a_{-}, a_{+}, b ; z\right)$ and $\alpha_{n}\left(a_{+}, a_{-}, b ; z\right)$ may be replaced by $b \mapsto b+\left(2 N_{-}+1\right) a_{-}$, one obtains $(2.36)$ for $b=\left(N_{+}+1\right) a_{+}+\left(N_{-}+1\right) a_{-}$and $b=-N_{+} a_{+}-N_{-} a_{-}$, as well. (These equalities can also be obtained directly from the results in section 3 of [2].)

We proceed by observing that we can use $(2.29)$ to obtain a second independent formal power series $\mathcal{A}_{\delta}$-eigenfunction $F_{-}\left(a_{+}, a_{-}, b ; z, y\right)$. It also has the symmetry properties $(2.20),(2.21)$, since the $u$-function does. Furthermore, its interpolation properties follow from those of $F_{+}\left(a_{+}, a_{-}, b ; z, y\right)$, since $u\left(a_{+}, a_{-}, b ; z\right)$ admits a meromorphic interpolation.

The $c$-function has the latter feature, too, $\mathrm{cf}(1.20)$. Therefore, the interpolation behaviour of the function $M(z, p)$ is also equivalent to that of $F_{+}(z, y), \mathrm{cf}(2.27)$. We already made some remarks on the interpolation problem for $M(z, p)$ in [2]. (See also section 4.4 of our lecture notes [7].) Here we can add that (2.59) implies that the poles of the coefficients of the formal power series eigenfunctions are indeed dense on the imaginary $z$-axis whenever $b$ is not of the form $k a_{+}+l a_{-}, k, l \in \mathbb{Z}$. (This denseness property was already discussed in [1].) This state of affairs suggests that whenever a non-formal eigenfunction for such $b$-values exists, for which the formal eigenfunction $F_{+}(z, y)$ is an asymptotic series, it will have the imaginary $z$-axis as a natural boundary.

Let us finally consider the case of a rational quotient $a_{-} / a_{+}=M / N$, with $M$ and $N$ coprime integers. Then $\alpha_{n}\left(a_{+}, a_{-}, b ; z\right)$ is still well defined for $n<N$. Likewise, $\alpha_{m}\left(a_{-}, a_{+}, b ; z\right)$ has no divergencies for $m<M$. But for larger $n$-or $m$-values the coefficients are divergent, which reflects the equality of (for instance) the monomials $s_{+}^{M}$ and $s_{-}^{N}, \operatorname{cf}(2.42)$. On the other hand, it may well be that the pertinent confluence limits are finite. This hunch is suggested by its validity for the simplest case, which is the limit $a_{+} \rightarrow a_{-}$for the lowest order monomials. Indeed, using (2.61), one easily checks that

$$
\lim _{a_{+} \rightarrow a_{-}}\left(\alpha_{1}\left(a_{+}, a_{-}, b ; z\right) \mathrm{e}^{-2 a_{-} y}+\alpha_{1}\left(a_{-}, a_{+}, b ; z\right) \mathrm{e}^{-2 a_{+} y}\right)
$$

exists.

\section{The elliptic regime}

\subsection{A modified power series ansatz: the case $m+n<3$}

In the elliptic case the results from [1] already mentioned in the introduction give rise to joint eigenvalues $E_{ \pm}(y)$ that have asymptotics (1.32). But only in some special cases do these eigenvalues equal $2 \cosh \left(a_{\mp} y\right)$. Even so, we may attempt to follow the reasoning in section 2.1 , eigenfunctions of the latter eigenvalues, of (2.1). That is, we may try and obtain formal joint elliptic $A \Delta O s(1.10),(1.27)$. Doing so, we obtain once more the system (2.6)-(2.8). In the elliptic case, however, we
should clearly drop the asymptotics assumption (2.11). Thus, $(2.14)$ should be omitted as 
well. But as will now be shown, there does not even exist a function $\alpha_{1}$ satisfying both (2.12) and (2.13). As a preliminary, we note that (1.29) entails the $\mathrm{A} \Delta \mathrm{E}$

$$
\frac{s(r, a ; z+\mathrm{i} a / 2)}{s(r, a ; z-\mathrm{i} a / 2)}=-\exp (-2 \mathrm{i} r z)
$$

whence one has

$$
\frac{s^{\prime}(r, a ; z-\mathrm{i} a)}{s(r, a ; z-\mathrm{i} a)}-\frac{s^{\prime}(r, a ; z)}{s(r, a ; z)}=2 \mathrm{i} r .
$$

In order to prove the asserted non-existence, we consider the $\mathrm{A} \Delta \mathrm{E}(2.22)$ with $C_{+}(z)$ given by (1.27). The elliptic function $C_{+}(z)$ has two simple poles in its period rectangle, so the sum of the two residues vanishes. Therefore one can uniquely write $C_{+}(z)$ as

$$
C_{+}(z)=A\left(a_{+}, a_{-}\right)+B\left(a_{+}, a_{-}\right)\left(\frac{s_{+}^{\prime}\left(z+\mathrm{i} a_{-}\right)}{s_{+}\left(z+\mathrm{i} a_{-}\right)}-\frac{s_{+}^{\prime}(z)}{s_{+}(z)}\right) .
$$

Comparing residues at $x=-\mathrm{i} a_{-}$yields

$$
B\left(a_{+}, a_{-}\right)=\mathrm{e}^{-2 b r} s_{+}\left(\mathrm{i} b-\mathrm{i} a_{-}\right) s_{+}(\mathrm{i} b) / s_{+}\left(\mathrm{i} a_{-}\right) .
$$

Taking now $z=-\mathrm{i} b$, one obtains

$$
A\left(a_{+}, a_{-}\right)=\frac{\mathrm{e}^{-2 b r}}{s_{+}\left(\mathrm{i} a a_{-}\right)}\left[s_{+}(\mathrm{i} b) s_{+}^{\prime}\left(\mathrm{i} b-\mathrm{i} a_{-}\right)-s_{+}^{\prime}(\mathrm{i} b) s_{+}\left(\mathrm{i} b-\mathrm{i} a_{-}\right)\right] .
$$

Defining $\rho(z)$ by

$$
\alpha_{1}(z)=B \frac{s_{+}^{\prime}\left(z+\mathrm{i} a_{-}\right)}{s_{+}\left(z+\mathrm{i} a_{-}\right)}+\rho(z)
$$

the $\mathrm{A} \Delta \mathrm{E}(2.22)$ and requirement $\alpha_{1} \in \mathcal{P}_{\mathrm{i} a_{+}}$now yield

$$
\begin{aligned}
& \rho\left(z-\mathrm{i} a_{-}\right)-\rho(z)=1-A \\
& \rho\left(z-\mathrm{i} a_{+}\right)-\rho(z)=B\left(\frac{s_{+}^{\prime}\left(z+\mathrm{i} a_{-}\right)}{s_{+}\left(z+\mathrm{i} a_{-}\right)}-\frac{s_{+}^{\prime}\left(z+\mathrm{i} a_{-}-\mathrm{i} a_{+}\right)}{s_{+}\left(z+\mathrm{i} a_{-}-\mathrm{i} a_{+}\right)}\right)=-2 \mathrm{i} r B
\end{aligned}
$$

where we used (3.2).

Taking the $z$-derivative of the two $\mathrm{A} \Delta \mathrm{Es}$ (3.7) and (3.8), we obtain $\rho^{\prime}(z)=c$ from (1.12). Thus we have $\rho(z)=c z+d$, with

$$
-\mathrm{i} a_{-} c=1-A \quad-\mathrm{i} a_{+} c=-2 \mathrm{i} r B .
$$

Now from (3.5) and (3.4) we see that $A \neq 1$ and $B \neq 0$. Hence we need

$$
1-A=-2 \mathrm{ir} a_{-} B / a_{+} \text {. }
$$

But in view of (3.4) and (3.5) this relation only holds for special $b$-values (in particular, for $b=0$.)

We are therefore led to an illuminating conclusion: Even at the level of formal power series, the ansatz (2.4) is not compatible with $\mathcal{A}_{\delta}$-eigenvalues $2 \cosh \left(a_{-\delta} y\right), \delta=+,-$.

On the other hand, we may consider a power series ansatz for the eigenvalues, too. Specifically, let us assume

$E_{\delta}(y)=\exp \left(a_{-\delta} y\right) \sum_{m, n=0}^{\infty} e_{m n}^{\delta} \exp \left(-2\left(m a_{+}+n a_{-}\right) y\right) \quad e_{00}^{\delta}=1 \quad \delta=+,-$.

(Hence (1.32) formally follows.) As the generalization of (2.6) and (2.7) we then get

$$
\begin{array}{ll}
c_{m n}\left(z-\mathrm{i} a_{+}\right)-c_{m n}(z)=-C_{-}(z) c_{m-1, n}\left(z+\mathrm{i} a_{+}\right)+\sum_{k, l}^{m, n} e_{m-k, n-l}^{-} c_{k l}(z) & m, n \in \mathbb{N} \\
c_{m n}\left(z-\mathrm{i} a_{-}\right)-c_{m n}(z)=-C_{+}(z) c_{m, n-1}\left(z+\mathrm{i} a_{-}\right)+\sum_{k, l}^{m, n} e_{m-k, n-l}^{+} c_{k l}(z) & m, n \in \mathbb{N} .
\end{array}
$$


Here, the summation symbol signifies a sum over $k \in\{0,1, \ldots, m\}$ and $l \in\{0,1, \ldots, n\}$, omitting the term $(k, l)=(m, n)$.

Taking first $m=0, n=1$ in this recursive system of A $\Delta \mathrm{Es}$, we obtain

$$
\begin{aligned}
& c_{01}\left(z-\mathrm{i} a_{+}\right)-c_{01}(z)=e_{01}^{-} \\
& c_{01}\left(z-\mathrm{i} a_{-}\right)-c_{01}(z)=-C_{+}(z)+e_{01}^{+} .
\end{aligned}
$$

Using as before (3.3)-(3.5), we write

$$
c_{01}(z)=B \frac{s_{+}^{\prime}\left(z+\mathrm{i} a_{-}\right)}{s_{+}\left(z+\mathrm{i} a_{-}\right)}+\rho(z)
$$

so that $\rho(z)$ satisfies

$$
\begin{aligned}
& \rho\left(z-\mathrm{i} a_{-}\right)-\rho(z)=-A+e_{01}^{+} \\
& \rho\left(z-\mathrm{i} a_{+}\right)-\rho(z)=-2 \mathrm{ir} B+e_{01}^{-} .
\end{aligned}
$$

Thus we get $\rho(z)=c z+d$.

At this stage we cannot rule out that $c$ is non-zero, since we are allowing quite general eigenvalues. But our principal interest is in coefficients $c_{m n}(z)$ that are $\pi / r$-periodic in $z$. This is because the joint eigenfunction $\Psi(z, y)$ from [1] has the property that $\Psi(z, y) \exp (-i z y)$ is $\pi / r$-periodic in $z$; since $c(z)(1.31)$ is $\pi / r$-periodic, too, it follows that $F_{+}(z, y) \exp (-\mathrm{i} z y)$ is $\pi / r$-periodic, $\operatorname{cf}(1.24)$.

Accordingly, we require from now on

$$
c_{m n}(z) \in \mathcal{P}_{\pi / r} \quad m, n \in \mathbb{N} .
$$

The above then yields

$$
\begin{aligned}
& c_{01}(z)=B\left(a_{+}, a_{-}\right) \frac{s_{+}^{\prime}\left(z+\mathrm{i} a_{-}\right)}{s_{+}\left(z+\mathrm{i} a_{-}\right)}+\gamma_{01} \\
& e_{01}^{-}=2 \mathrm{i} r B\left(a_{+}, a_{-}\right) \\
& e_{01}^{+}=A\left(a_{+}, a_{-}\right)
\end{aligned}
$$

with $A\left(a_{+}, a_{-}\right)$and $B\left(a_{+}, a_{-}\right)$given by (3.5) and (3.4), resp. In particular, this entails that $E_{\delta}(y)$ depends on all of the parameters $r, a_{+}, a_{-}$and $b$, in contrast to the hyperbolic case. The constant $\gamma_{01}$ cannot be fixed unless we impose further restrictions on $c_{01}(z)$. In this connection we recall the ambiguity pointed out in the paragraph containing $(2.10)$. Now at the elliptic level there is no natural generalization of the asymptotics requirement (2.11). Thus we should try and fix the dependence on $y$ in some other way.

We will return to the $y$-dependence shortly, but we allow the ambiguity for the time being. Clearly, we can repeat our reasoning for the equations

so that we obtain

$$
\begin{aligned}
& c_{10}\left(z-\mathrm{i} a_{-}\right)-c_{10}(z)=e_{10}^{+} \\
& c_{10}\left(z-\mathrm{i} a_{+}\right)-c_{10}(z)=-C_{-}(z)+e_{10}^{-}
\end{aligned}
$$

$$
\begin{aligned}
& c_{10}(z)=B\left(a_{-}, a_{+}\right) \frac{s_{-}^{\prime}\left(z+\mathrm{i} a_{+}\right)}{s_{-}\left(z+\mathrm{i} a_{+}\right)}+\gamma_{10} \\
& e_{10}^{+}=2 \mathrm{ir} B\left(a_{-}, a_{+}\right) \\
& e_{10}^{-}=A\left(a_{-}, a_{+}\right) .
\end{aligned}
$$

Consider next $c_{11}$. We should solve the ADEs

$$
\begin{aligned}
& c_{11}\left(z-i a_{+}\right)-c_{11}(z)=-C_{-}(z) c_{01}\left(z+i a_{+}\right)+e_{11}^{-}+e_{10}^{-} c_{01}(z)+e_{01}^{-} c_{10}(z) \\
& c_{11}\left(z-i a_{-}\right)-c_{11}(z)=-C_{+}(z) c_{10}\left(z+i a_{-}\right)+e_{11}^{+}+e_{10}^{+} c_{01}(z)+e_{01}^{+} c_{10}(z)
\end{aligned}
$$


for a $\pi / r$-periodic function $c_{11}(z)$. By the same reasoning as before, when such a solution $c_{11}(z)$ exists, then it is unique up to a constant, while $e_{11}^{-}$and $e_{11}^{+}$are uniquely determined. Using (3.15) and (3.24) to get rid of $C_{ \pm}(z)$, one easily verifies that the desired solution reads

$$
c_{11}(z)=\left[c_{10}(z)-2 e_{10}^{+}\right]\left[c_{01}(z)-2 e_{01}^{-}\right]+\gamma_{11}
$$

yielding

$$
\begin{aligned}
& e_{11}^{-}=-e_{01}^{-}\left(e_{10}^{-}+2 e_{10}^{+}\right) \\
& e_{11}^{+}=-e_{10}^{+}\left(e_{01}^{+}+2 e_{01}^{-}\right) .
\end{aligned}
$$

Before discussing the general situation, we obtain a few more quantities explicitly, namely $c_{02}, e_{02}^{\delta}, c_{20}, e_{20}^{\delta}, \delta=+,-$. The first one should be a $\pi / r$-periodic function satisfying the $\mathrm{A} \triangle \mathrm{ES}$

$$
\begin{aligned}
& c_{02}\left(z-\mathrm{i} a_{+}\right)-c_{02}(z)=e_{02}^{-}+e_{01}^{-} c_{01}(z) \\
& c_{02}\left(z-\mathrm{i} a_{-}\right)-c_{02}(z)=\left[c_{01}\left(z-\mathrm{i} a_{-}\right)-c_{01}(z)-e_{01}^{+}\right] c_{01}\left(z+\mathrm{i} a_{-}\right)+e_{02}^{+}+e_{01}^{+} c_{01}(z) .
\end{aligned}
$$

As before, any function with these features is unique up to a constant.

We claim such a function exists and is given by

$$
\begin{aligned}
& c_{02}(z)=\frac{B\left(a_{+}, a_{-}\right)^{2}}{2} \frac{s_{+}^{\prime \prime}\left(z+\mathrm{i} a_{-}\right)}{s_{+}\left(z+\mathrm{i} a_{-}\right)}+\zeta_{1} c_{01}(z)+\zeta_{2} c_{01}\left(z+\mathrm{i} a_{-}\right)+\gamma_{02} \\
& \zeta_{1} \equiv c_{01}\left(\mathrm{i} a_{-}\right) \quad \zeta_{2} \equiv e_{01}^{+}-c_{01}(0)+c_{01}\left(\mathrm{i} a_{-}\right) .
\end{aligned}
$$

Indeed, the rhs is clearly $\pi / r$-periodic. To verify the $\mathrm{A} \Delta \mathrm{E}$ (3.33), one needs the identity

$$
\frac{s_{+}^{\prime \prime}\left(z-\mathrm{i} a_{+}\right)}{s_{+}\left(z-\mathrm{i} a_{+}\right)}-\frac{s_{+}^{\prime \prime}(z)}{s_{+}(z)}=4 \mathrm{i} r \frac{s_{+}^{\prime}(z)}{s_{+}(z)}-4 r^{2}
$$

which follows by differentiating (3.2). To check (3.34), one can proceed as follows. First, although the difference $\Delta(z)$ between the lhs and rhs consists of terms that are not i $a_{+}$-periodic, $\Delta(z)$ is in fact $i a_{+}$-periodic. (This can be verified by using (3.2) and (3.37).) Therefore $\Delta(z)$ is elliptic. Requiring now that the residues at the simple poles $z=0$ and $z=-2 \mathrm{i} a$ - cancel, one obtains (3.36). Then the residues at $z=-\mathrm{i} a_{-}$cancel, too, so $\Delta(z)$ is $z$-independent.

Choosing $z=-\mathrm{i} b$ in $\Delta(z)$ so as to exploit $C_{+}(-\mathrm{i} b)=0(\mathrm{cf}(1.27))$, one now obtains

$$
\begin{gathered}
e_{02}^{+}=\frac{B\left(a_{+}, a_{-}\right)^{2}}{2}\left(\frac{s_{+}^{\prime \prime}(\mathrm{i} b)}{s_{+}(\mathrm{i} b)}-\frac{s_{+}^{\prime \prime}\left(\mathrm{i} b-\mathrm{i} a_{-}\right)}{s_{+}\left(\mathrm{i} b-\mathrm{i} a_{-}\right)}\right)+e_{01}^{+}\left[c_{01}\left(\mathrm{i} a_{-}\right)-c_{01}\left(-\mathrm{i} b+\mathrm{i} a_{-}\right)\right] \\
+\left[c_{01}\left(\mathrm{i} a_{-}\right)-c_{01}(0)\right]\left[c_{01}(-\mathrm{i} b)-c_{01}\left(-\mathrm{i} b+\mathrm{i} a_{-}\right)\right] .
\end{gathered}
$$

Likewise, from (3.33) one gets

$e_{02}^{-}=B\left(a_{+}, a_{-}\right)^{2}\left(2 \mathrm{i} r \frac{s_{+}^{\prime}\left(2 \mathrm{i} a_{-}\right)}{s_{+}\left(2 \mathrm{i} a_{-}\right)}-2 r^{2}\right)+e_{01}^{-}\left[c_{01}\left(\mathrm{i} a_{-}\right)-c_{01}(0)+e_{01}^{+}\right]$

Clearly, we can now determine $c_{20}(z)$ and $e_{20}^{\delta}, \delta=+,-$, by switching indices and $a_{+}, a_{-}$. Therefore, all quantities $c_{m n}(z), e_{m n}^{\delta}$ with $m+n \leqslant 2$ have now been obtained in explicit form.

\subsection{An appraisal of the general case}

We proceed with a study of the general situation. Obviously, we can analyse the system (3.12), (3.13) with boundary conditions (2.8) recursively, the conclusion being that when a solution satisfying (3.19) exists, then it is unique up to the ambiguity (2.10), whereas the formal power series (3.11) have uniquely determined coefficients. Now in the hyperbolic case we could exploit our previous results from [2] to prove that for $b=\left(N_{+}+1\right) a_{+}-N_{-} a_{-}$ 
the system (2.6)-(2.8) does admit a solution. Although the results in [1] suggest that this is still true for the pertinent elliptic system, we have no complete proof.

In order to explain in more detail what is involved here, it is expedient to consider first two cases for which existence of a solution does follow from [1]. Specifically, these are the cases $b=\left(N_{+}+1\right) a_{+}$and $b=\left(N_{-}+1\right) a_{-}$, with $N_{\delta} \in \mathbb{N}$. (These special cases were also studied in $[8,9]$.) By $a_{+} \leftrightarrow a_{-}$symmetry, we need only discuss the first case.

First, we recall from section 2 in [1] that the function

$$
F_{+}(z, y) \equiv c(z)^{-1} \Psi(z, y)=\mathrm{e}^{\mathrm{i} z y} \prod_{j=1}^{N_{+}} \frac{s_{-}\left(z+z_{j}(y)\right)}{s_{-}\left(z+\mathrm{i} j a_{+}\right)} \quad y \in(K, \infty)
$$

is an $\mathcal{A}_{\delta}$-eigenfunction with eigenvalues $E_{\delta}(y), \delta=+,-$. (We are choosing the function $f(y)$ in (1.24) equal to 1 , since we have no other natural 'dual choice' available.) The point is now that the solutions to the constraint system detailed in [1] admit a convergent power series expansion

$$
z_{j}(y)=\sum_{k=0}^{\infty} \eta_{j, k} \exp \left(-2 k a_{+} y\right) \quad \eta_{j, 0} \equiv \mathrm{i} j a_{+} \quad j=1, \ldots, N_{+}
$$

for $y$ large enough.

Indeed, from the paragraph in [1] containing equation (2.15) we deduce that the parameters $y$ and $t$ are related by $\exp \left(-2 a_{+} y\right)=f(t)$, where $f(t)$ is holomorphic at $t=0$ and satisfies $f(0)=0, f^{\prime}(0) \neq 0$. Therefore, there exists a function $H(z)$ that is holomorphic at $z=0$ and satisfies $H(0)=0, H^{\prime}(0) \neq 0$, such that $t=H\left(\exp \left(-2 a_{+} y\right)\right)$. Hence we obtain $(3.41)$.

Using also equations $(2.16)$ and $(2.27)$ in [1] we now deduce that there exist convergent
ansions expansions

$$
\begin{array}{ll}
\exp (-\mathrm{i} z y) F_{+}(z, y)=\sum_{k=0}^{\infty} c_{k 0}(z) \exp \left(-2 k a_{+} y\right) & c_{00}=1 \\
\exp \left(-a_{+} y\right) E_{-}(y)=\sum_{k=0}^{\infty} e_{k 0}^{-} \exp \left(-2 k a_{+} y\right) & e_{00}^{-}=1 \\
\exp \left(-a_{-} y\right) E_{+}(y)=\sum_{k=0}^{\infty}\left[e_{k 0}^{+}+e_{k 1}^{+} \exp \left(-2 a_{-} y\right)\right] \exp \left(-2 k a_{+} y\right) \quad e_{00}^{+}=1
\end{array}
$$

for $y$ large enough. In particular, this entails

$$
\begin{array}{lllll}
c_{k l}(z)=0 & e_{k l}^{-}=0 & e_{k, l+1}^{+}=0 & k \in \mathbb{N} & l \in \mathbb{N}^{*} \quad\left(b=\left(N_{+}+1\right) a_{+}\right)
\end{array}
$$

in accordance with the special cases calculated in section 3.1.
Next, we study the dense set of $b$-values $\left.\left(N_{+}+1\right)\right)_{+}$

to the hyperbolic case, we are not $b$-values $\left(N_{+}+1\right) a_{+}-N_{-} a_{-}, N_{+}, N_{-} \in \mathbb{N}^{*}$. In contrast with boundary conditions (2.8) and periodicity complete proof that the system (3.12), (3.13) these $b$-values. To explain the problem that ariogirement (3.19) admits a solution for all of equations (1.21)-(1.25) in [1], we deduce thises, we first recall some results from [1]. From

$$
F_{+}(z, y) \equiv c(z)^{-1} \Psi(z, y)=\mathrm{e}^{\mathrm{i} z y} \prod_{\delta=+,-j=1} \prod_{j}^{N_{\beta}} \frac{s_{-\delta}\left(z+z_{j}^{\delta}(y)\right)}{s_{-\delta}\left(z+i j a_{8}\right)} \quad y \in(K, \infty)
$$
is an $\mathcal{A}_{\delta}$-eigenfunction with eigenvalues $E_{\delta}(y), \delta=+,-$. (Thus we have once again $f(y)=1$
in (1.24).) The question is now whether the solutions $z_{j}^{\delta}(y)$ to the constraint system detailed 
in section 3 of [1] admit a convergent power series expansion

$z_{j}^{\delta}(y)=\sum_{k, l=0}^{\infty} \eta_{j, k l}^{\delta} \exp \left(-2\left(k a_{+}+l a_{-}\right) y\right) \quad \eta_{j, 00}^{\delta} \equiv i j a_{\delta} \quad j=1, \ldots, N_{\delta} \quad \delta=+,-$

for $y$ large enough. If so, this would follow for $\exp (-\mathrm{i} z y) F_{+}(z, y)$ and $\exp \left(-a_{-\delta} y\right) E_{\delta}(y)$, as well.

Elaborating on the latter question, we observe that the parameters $u$ and $t_{\delta}$ introduced in [1] are related via $\exp \left(-2 a_{\delta} u\right)=f_{\delta}\left(t_{\delta}\right)$, where $f_{\delta}(z)$ is holomorphic at $z=0$ and satisfies $f_{\delta}(0)=0, f_{\delta}^{\prime}(0) \neq 0$. (This follows from equation (3.16) in [1] by writing the lhs as $u$, and then exponentiating.) Therefore, there exist two functions $H_{ \pm}(z)$ that are holomorphic at $z=0$ and satisfy $H_{ \pm}(0)=0, H_{ \pm}^{\prime}(0) \neq 0$, such that $t_{ \pm}=H_{ \pm}\left(\exp \left(-2 a_{ \pm} u\right)\right)$. From [1] equation (3.17) we now deduce that we have $y=u+H\left(\exp \left(-2 a_{+} u\right), \exp \left(-2 a_{-} u\right)\right)$, with $H\left(z_{1}, z_{2}\right)$ holomorphic at $z_{1}=z_{2}=0$. But we do not know whether an 'inverse' function $I\left(z_{1}, z_{2}\right)$ exists, holomorphic at $z_{1}=z_{2}=0$ and such that $u=y+I\left(\exp \left(-2 a_{+} y\right), \exp \left(-2 a_{-} y\right)\right)$. If so, we would have

$$
\exp \left(-2 a_{\delta} u\right)=\exp \left(-2 a_{\delta} y\right) \sum_{k, l=0}^{\infty} \tau_{k l}^{\delta} \exp \left(-2\left(k a_{+}+l a_{-}\right) y\right)
$$

with the power series convergent for $y$ large enough. Thus it would follow that $z_{j}^{\delta}(y)$, hence $\exp \left(-a_{-\delta} y\right) E_{\delta}(y)$ and $\exp (-\mathrm{i} z y) F_{+}(z, y)$ as well, admit convergent power series expansions in $\exp \left(-2 a_{+} y\right)$ and $\exp \left(-2 a_{-} y\right)$ for $y$ large enough.

Even when the above question admits an affirmative answer (as we expect), it appears an intractable task to actually calculate the coefficients explicitly by taking $F_{+}(z, y)(3.46)$ as a starting point. In the hyperbolic case we could invoke the second, far more explicit representation (2.36) of the joint eigenfunction, which enabled us to bypass the highly transcendental dependence of the zero functions $z_{j}^{\delta}(y)$ on $y$. But we are not aware of any alternative representations in the elliptic case, so that a further analysis of the system (3.12), (3.13) with boundary conditions (2.8) and periodicity requirement (3.19) appears more promising.

Even though we are unable to prove that the latter system admits solutions for all of the $b$-values $\left(N_{+}+1\right) a_{+}-N_{-} a_{-}$, we should repeat that we have shown above that no obstructions occur for the cases $m+n \leqslant 2$. Moreover, all of the pertinent quantities in section 3.1 are real-analytic in $b$ for real $b$. This strongly suggests that formal interpolating solutions with essentially the same features as in the hyperbolic case do exist.

\section{References}

[1] Ruijsenaars S N M 1999 Generalized Lamé functions. I. The elliptic case J. Math. Phys. 40 1595-626

[2] Ruijsenaars S N M 1999 Generalized Lame functions. II. Hyperbolic and trigonometric specializations J. Math. Phys. $401627-63$

[3] Ruijsenaars SNM 2000 Special functions defined by analytic difference equations Proc. Tempe NATOAdvanced Study Institute 'Special Functions 2000' ed J Bustoz, M Ismail, S K Suslov, at press

[4] Ruijsenaars S N M 1997 First-order analytic difference equations and integrable quantum systems J. Math. Phys. 38 1069-146

[5] van Diejen J F and Kirillov A N 2000 Formulae for $q$-spherical functions using inverse scattering theory of reflectionless Jacobi operators Commun. Math. Phys. $210335-69$

[6] Gasper G and Rahman M 1990 Basic hypergeometric series Encyclopedia of Mathematics and its Applications vol 35 (Cambridge: Cambridge University Press)

[7] Ruijsenaars S N M 2000 Special functions associated with Calogero-Moser type quantum systems Proc. 1999 Montreal SEminaire de Mathematiques Superieures (CRM Proceedings and Lecture Notes vol 26) ed J Harnad, G Sabidussi and P Winternitz (Providence, RI: American Mathematical Society) pp 189-226 
[8] Krichever I and Zabrodin A 1995 Spin generalization of the Ruijsenaars-Schneider model, non-Abelian 2D Toda chain and representations of Sklyanin algebra Russ. Math. Surv. $501101-50$

[9] Felder $G$ and Varchenko $A 1996$ Algebraic Bethe ansatz for the elliptic quantum group $E_{\tau, \eta}\left(s l_{2}\right) N u c l$. Phys. B $480485-503$ 